\title{
Optical loss and lasing characteristics of high-quality-factor AIGaAs microdisk resonators with embedded quantum dots
}

\author{
Kartik Srinivasan, ${ }^{\text {a) }}$ Matthew Borselli, Thomas J. Johnson, \\ Paul E. Barclay, and Oskar Painter \\ Thomas J. Watson, Sr. Laboratory of Applied Physics, California Institute of Technology, Pasadena, \\ California 91125 \\ Andreas Stintz and Sanjay Krishna \\ Center for High Technology Materials, University of New Mexico, Albuquerque, New Mexico 87106
}

(Received 16 December 2004; accepted 28 February 2005; published online 6 April 2005)

\begin{abstract}
Optical characterization of $\mathrm{AlGaAs}$ microdisk resonant cavities with a quantum dot active region is presented. Direct passive measurement of the optical loss within AlGaAs microdisk resonant structures embedded with InAs/InGaAs dots-in-a-well (DWELL) is performed using an optical-fiber-based probing technique at a wavelength $(\lambda \sim 1.4 \mu \mathrm{m})$ that is red detuned from the dot emission wavelength $(\lambda \sim 1.2 \mu \mathrm{m})$. Measurements in the $1.4 \mu \mathrm{m}$ wavelength band on microdisks of diameter $D=4.5 \mu \mathrm{m}$ show that these structures support modes with cold-cavity quality factors as high as $3.6 \times 10^{5}$. DWELL-containing microdisks are then studied through optical pumping at room temperature. Pulsed lasing at $\lambda \sim 1.2 \mu \mathrm{m}$ is seen for cavities containing a single layer of InAs dots, with threshold values of $\sim 17 \mu \mathrm{W}$, approaching the estimated material transparency level. Room-temperature continuous-wave operation is also observed. (c) 2005 American Institute of Physics. [DOI: 10.1063/1.1901810]
\end{abstract}

Recently, multiple research groups have demonstrated vacuum Rabi splitting in a semiconductor system consisting of a single quantum-dot (QD) exciton embedded in an optical microcavity. ${ }^{1-3}$ These experiments have in many ways confirmed the potential of semiconductor microcavities for chip-based cavity quantum electrodynamics (cQED) experiments. For future experiments, such as those involving quantum state transfer in quantum networks, ${ }^{4}$ it will be important to further improve upon the parameters of such QDmicrocavity systems. One clear improvement required is to move the system further within the regime of strong coupling, ${ }^{5}$ defined as having the atom-photon coupling rate $(g)$ larger than both the cavity loss rate $(\kappa)$ and QD dipole decay rate $(\gamma)$. In particular, the ratio of $g$ to the larger of $\kappa$ and $\gamma$ approximately represents the number of Rabi oscillations that can take place before the effects of dissipation destroy coherent energy exchange. ${ }^{5}$ In each of Refs. $1-3$, loss in the system was found to be dominated by the optical cavity, with $g \sim \kappa$. As the low-temperature homogeneous linewidth in self-assembled InAs QDs is typically a few $\mu \mathrm{eV},{ }^{6}$ corresponding to a QD dipole decay rate of $\gamma / 2 \pi \sim 1 \mathrm{GHz}$, it will be advantageous to develop cavities with quality factors such that $\kappa / 2 \pi \leqslant 1 \mathrm{GHz}$, with further improvements in $Q$ serving mainly to improve the optical collection efficiency of emitted light. For the $\lambda \sim 0.9-1.2 \mu \mathrm{m}$ emission wavelength for InAs QDs, ${ }^{1,2}$ this corresponds to an optical mode quality factor of $Q \sim 1 \times 10^{5}(\kappa / 2 \pi=\omega / 4 \pi Q)$. Achieving such low loss cavities is also important in light of the difficulty in fabricating a structure where the QD is optimally positioned for maximum coupling to the cavity mode.

Alongside the work on CQED in QD-microcavity systems, there has been significant progress in developing higher $Q$ and smaller effective mode volume $\left(V_{\text {eff }}\right)$ semicon-

\footnotetext{
${ }^{a)}$ Electronic mail: kartik@caltech.edu
}

ductor microcavities over the last few years. A variety of geometries and materials have been studied, ranging from InP photonic crystal microcavities $\left(Q \sim 1.3 \times 10^{4}, V_{\text {eff }}\right.$ $\left.\sim(\lambda / n)^{3}\right) \quad\left(\right.$ Ref. 7) to $\mathrm{Si}$ photonic crystal $^{8,9}[Q \sim 4$ $\left.\times 10^{4}, V_{\text {eff }} \sim(\lambda / n)^{3}\right]$ and microdisk ${ }^{10} \quad\left[Q \sim 5 \times 10^{5}, V_{\text {eff }}\right.$ $\left.\sim 5(\lambda / n)^{3}\right]$ cavities. Of particular importance to the selfassembled InAs QD work is the host AlGaAs material system. Here, we report the creation of $D=4.5 \mu \mathrm{m}$ diameter AlGaAs microdisks that exhibit $Q$ factors as high as 3.6 $\times 10^{5}$ at $\lambda \sim 1.4 \mu \mathrm{m}$, a value which exceeds the highest- $Q$ factors measured for AlGaAs microcavities. ${ }^{1,2,11,12}$ These AlGaAs microdisks contain embedded quantum dots-in-awell (DWELL) (Refs. 13 and 14) which have a ground-state emission at $\lambda \sim 1.2 \mu \mathrm{m}$, so the passive measurements at $\lambda$ $\sim 1.4 \mu \mathrm{m}$ are performed where the QDs are relatively nonabsorbing. The lasing characteristics of these devices are also investigated through photoluminescence measurements, and low threshold room-temperature QD lasers are demonstrated.

The microdisks studied here are fabricated from an epitaxy consisting of one or three layers of InAs QDs embedded in (1-3) $\mathrm{In}_{0.15} \mathrm{Ga}_{0.85} \mathrm{As}$ quantum wells, which are in turn sandwiched between $\mathrm{GaAs} / \mathrm{Al}_{0.3} \mathrm{Ga}_{0.7}$ As layers to create a total waveguide layer that is $d=255 \mathrm{~nm}$ thick. This layer is grown on top of a $1.5 \mu \mathrm{m} \mathrm{Al} \mathrm{Al}_{0.7} \mathrm{Ga}_{0.3}$ As sacrificial layer that forms the supporting pedestal [Fig. 1(c)]. The cavities are created through: (i) Electron-beam (e-beam) lithography and subsequent reflow of the e-beam resist to produce smooth and circular patterns, (ii) $\mathrm{SF}_{6} / \mathrm{C}_{4} \mathrm{~F}_{8}$ inductively coupled plasma reactive ion etching (ICP-RIE) of a deposited $\mathrm{Si}_{x} \mathrm{~N}_{y}$ mask layer [Fig. 1(a)], (iii) $\mathrm{Ar}^{-\mathrm{Cl}_{2}}$ ICP-RIE etching of the $\mathrm{Al}_{0.3} \mathrm{Ga}_{0.7}$ As layer and removal of the remaining $\mathrm{Si}_{x} \mathrm{~N}_{y}$ layer, and (iv) wet chemical etching of the underlying $\mathrm{Al}_{0.7} \mathrm{Ga}_{0.3} \mathrm{As}$ layer to form the supporting pedestal [Figs. 1(b) and 1(c)]. The $\mathrm{Si}_{x} \mathrm{~N}_{y}$ etch step is particularly important, as any roughness in this mask layer is transferred into underlying layers. This etch has thus been calibrated to produce as smooth a 


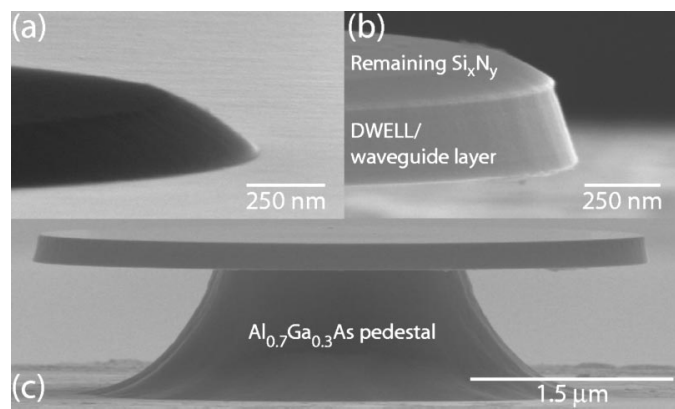

FIG. 1. Scanning electron microscope images of DWELL-containing microdisk cavities after the (a) $\mathrm{Si}_{x} \mathrm{~N}_{y}$ etch, and (b) and (c) AlGaAs etch and undercut.

sidewall surface as possible [Fig. 1(a)], without particular concern for its verticality. The subsequent $\mathrm{Ar}-\mathrm{Cl}_{2}$ etch is highly selective so that the angled mask does not result in erosion of the AlGaAs sidewalls.

Initial passive measurements to measure the cold-cavity $Q$ factor of the microdisk resonant modes were performed using an optical-fiber-based evanescent coupling technique. $9,10,15$ An optical fiber taper is formed by heating and adiabatically stretching a standard single-mode fiber until it reaches a minimum diameter $\sim 1 \mu \mathrm{m}$. A fiber-coupled scanning tunable laser $(<5 \mathrm{MHz}$ linewidth) is spliced to the taper's input, and when the taper is brought within a few hundred nanometers $(\mathrm{nm})$ of the cavity, their evanescent fields interact, and power transfer can result. A schematic illustrating the coupling geometry for this system is shown in Fig. 2(a). Further details of the mounting and positioning of the fiber taper are described in Refs. 9 and 10. The $Q$ of a cavity mode is determined by examining the linewidth of the resulting resonance in the taper's wavelength dependent transmission spectrum. In Fig. 2(b), we show a "doublet" resonance of a microdisk $(D=4.5 \mu \mathrm{m}$, one-DWELL structure) in the $1400 \mathrm{~nm}$ wavelength band when the taper is $\sim 800 \mathrm{~nm}$ to the side of the disk; the separation is kept large in order to reduce taper loading effects. ${ }^{9,10}$ The double resonance peaks correspond to standing wave modes formed from mixtures of the degenerate clockwise and counterclockwise whispering gallery modes that couple and split due to the disk-edge surface roughness. ${ }^{10,15,16}$ The linewidth $(\Delta \lambda)$ of the shorter wavelength resonance corresponds to $Q \sim 3.6$ $\times 10^{5}$. Similarly, in Fig. 2(c), we show the spectral response of the doublet when the taper is positioned much closer ( $\sim 200 \mathrm{~nm}$ ) to the edge of the disk, so that the amount of coupling has increased. The combination of increased coupling as well as parasitic loading due to the presence of the taper has increased the total loss rate of the resonant mode, yielding a loaded $Q \sim 1.0 \times 10^{5}$. The depth of coupling, however, has also considerably increased from $10 \%$ to $60 \%$, corresponding to a photon collection efficiency (the ratio of "good" coupling to all other cavity losses including parasitic and intrinsic modal loss) of approximately $20 \%$. It is believed that the high- $Q$ values achieved in these measurements are due to a combination of the resist reflow process that reduces radial variations and subsequent Rayleigh scattering in the disk, ${ }^{10}$ and the optimized dry etching processes that create very smooth disk-edge sidewalls.

The demonstrated $Q$ is high enough that, if used in cQED, the cavity will have a decay rate $\kappa / 2 \pi \sim 0.35 \mathrm{GHz}$ (at $\lambda \sim 1.2 \mu \mathrm{m}$ ), lower than the aforementioned typical low- mated threshold value of $\sim 22 \mu \mathrm{W}$.
Downloaded 14 Dec 2005 to 131.215.225.171. Redistribution subject to AlP license or copyright, see http://apl.aip.org/apl/copyright.jsp

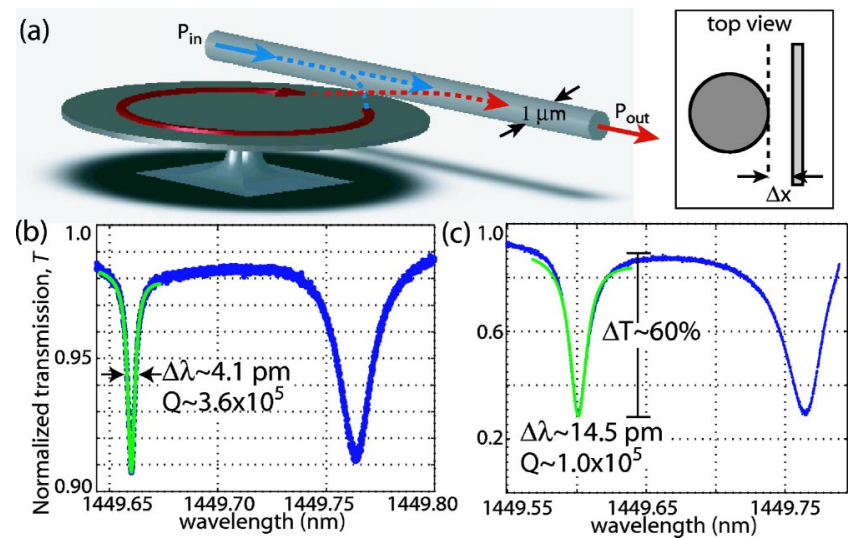

FIG. 2. (Color online). (a) Schematic geometry for probing the microdisk cavities through side coupling via an optical fiber taper. (b) Normalized taper transmission $\left(T=P_{\text {out }} / P_{\text {in }}\right)$ of a $4.5 \mu \mathrm{m}$ diameter microdisk for a taperdisk lateral separation $(\Delta x)$ of $\sim 800 \mathrm{~nm}$. (c) Normalized taper transmission for the same device with $\Delta x \sim 200 \mathrm{~nm}$. The overlying solid green curves are Lorentzian fits to the data.

temperature QD dipole decay rate of $\gamma / 2 \pi \sim 1 \mathrm{GHz}$. After adjusting for the reduced wavelength of the QD resonance, the current devices $(D=4.5 \mu \mathrm{m})$ have a $V_{\text {eff }} \sim 6(\lambda / n)^{3}$ for the standing wave resonant modes studied here. ${ }^{17}$ For a maximally coupled InAs QD (spontaneous emission lifetime $\tau \sim 1$ ns, oscillator strength $f \sim 18,{ }^{18}$ ) this mode volume corresponds to $g / 2 \pi \sim 11 \mathrm{GHz}$. Thus, even for the disk sizes considered in this work, an appropriately positioned QD would place the system deep within the strong coupling regime. Of additional importance is the fiber-based coupling technique used here. This method allows for the $Q$ to be accurately determined in a way that does not rely upon the (weak) background emission from the QDs; ${ }^{1-3}$ all that is required is a probe laser that can be slightly detuned from the QD absorption lines. Furthermore, the taper also acts as a coupler that transfers light from an optical fiber into the wavelength-scale mode volume of the cavity, where it can interact with the QDs, and as a subsequent output coupler. Such integration could markedly improve the collection efficiency in cQED experiments, particularly important for microdisk and photonic crystal cavities, which typically do not have a radiation pattern that can be effectively collected by free-space optics or a cleaved fiber. ${ }^{2}$

In addition to the fiber-based passive measurements of the microdisks at $\lambda \sim 1.4 \mu \mathrm{m}$, we performed roomtemperature photoluminescence measurements to study the QD emission in the $1.2 \mu \mathrm{m}$ wavelength band. The cavities ( $D=5 \mu \mathrm{m}$ in this case) were optically pumped at room temperature using a pulsed $830 \mathrm{~nm}$ semiconductor laser, and the emitted laser light was collected by a microscope objective and spectrally resolved in an optical spectrum analyzer containing three DWELLs due to their higher modal gain, roughly three times that of a single DWELL layer. ${ }^{14}$ Emission is observed for a few $(\sim 2-5)$ modes in a given microdisk [Fig. 3(a)], and the linewidths of the resonant modes (taken at sub-threshold pump powers) are as narrow as the resolution limit of the OSA [inset of Fig. 3(b)]. Figure 3(b) shows a typical light-in-light-out $(L-L)$ curve for a threeDWELL device pumped with a $300 \mathrm{~ns}$ period and $10 \mathrm{~ns}$ pulse width; the device exhibits lasing action with an estimated threshold value of $\sim 22 \mu \mathrm{W}$.

to AIP license or copyright, see http://apl.aip.org/apl/copyright.jsp (OSA). Initial measurements were performed on cavities 

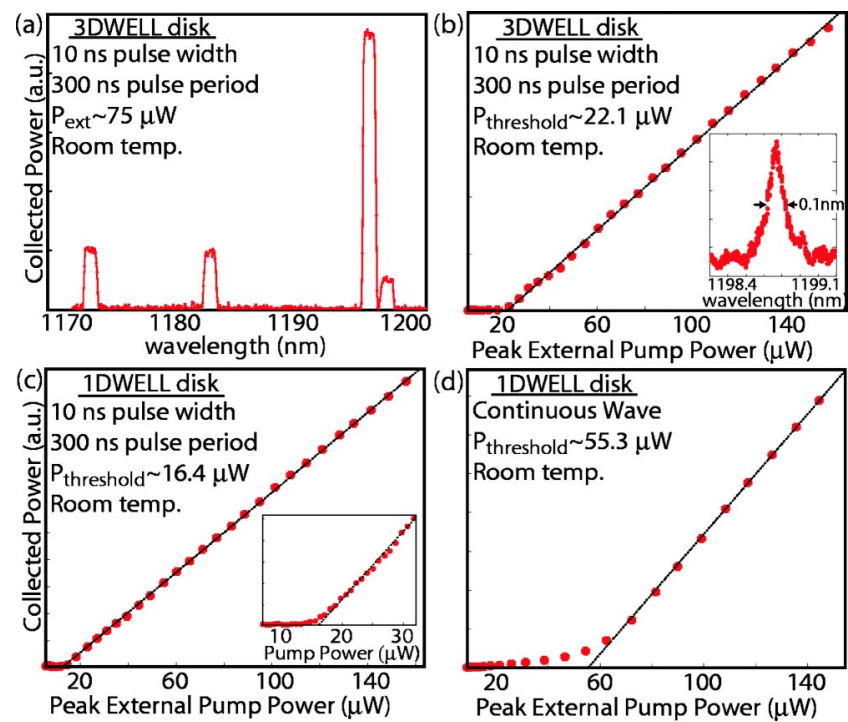

FIG. 3. (Color online). (a) Photoluminescence spectrum of a three-DWELL microdisk device [OSA resolution bandwidth $(\mathrm{RBW})=1 \mathrm{~nm}] .(\mathrm{b})-(\mathrm{d})$ $L-L$ curves for: (b) pulsed three-DWELL microdisk laser (inset shows the subthreshold spectrum of cavity mode with a resolution-limited (RBW $=0.10 \mathrm{~nm}$ ) linewidth), (c) pulsed one-DWELL microdisk laser (inset shows $L-L$ curve near threshold), and (d) one-DWELL microdisk laser under $\mathrm{cw}$ pumping conditions. The dashed lines are least-squares linear fits to the above-threshold data.

The saturated ground-state modal gain for single DWELL structures has been estimated to be $\sim 3.6-5.4 \mathrm{~cm}^{-1}{ }^{14,19}$ Noting that modal gain approximately equals the modal loss at threshold, this indicates that a minimum cavity $Q \sim 3-5 \times 10^{4}$ is required for this single layer of QDs to provide enough gain compensation to achieve lasing. The fiber-based linewidth measurements described earlier indicate that such $Q$ factors should be achievable, and indeed, lasing from the QD ground states is observed in these single dot layer devices [Fig. 3(c)]. The laser threshold pump power for the one-DWELL devices was measured to be as small as $16.4 \mu \mathrm{W}$, significantly lower than the $750 \mu \mathrm{W}$ threshold values recently reported for similarly sized microdisk QD lasers. ${ }^{20}$ Furthermore, as shown in Fig. 3(d), continuouswave $(\mathrm{cw})$ room-temperature lasing was also obtained, albeit with a somewhat higher laser threshold.

The laser threshold values we report here are the peak pump powers incident on the sample surface; the absorbed power is estimated to be roughly $16 \%$ of this value, determined by calculating the expected reflectivities at the disk interfaces and assuming an absorption coefficient of $10^{4} \mathrm{~cm}^{-1}$ in the GaAs and quantum-well layers. ${ }^{21}$ The threshold absorbed pump power for the one-DWELL lasers is thus $\sim 2.6 \mu \mathrm{W}$. From this, the equivalent threshold current density, useful for comparing the performance of the microdisk lasers to previously demonstrated broad-area stripe lasers, can be estimated. Given the pump spot size $\left(\sim 16 \mu \mathrm{m}^{2}\right)$, and assuming an internal quantum efficiency $\sim 1$, we arrive at an equivalent threshold current density of $11 \mathrm{~A} / \mathrm{cm}^{2}$ for the one-DWELL devices. In comparison, the estimated transparency current density in previous work on broad-area oneDWELL lasers was $10.1 \mathrm{~A} / \mathrm{cm}^{2}{ }^{14}$ The proximity of the demonstrated laser threshold to this transparency value indicates that nonintrinsic optical losses within the microdisk cavity have largely been eliminated.

In conclusion, AlGaAs microdisks as small as $4.5 \mu \mathrm{m}$ in diameter and supporting standing wave resonant modes with $Q$ factors as high as $3.6 \times 10^{5}$ in the $1400 \mathrm{~nm}$ wavelength band have been demonstrated. These cavities contain integral InAs quantum dots, and initial room-temperature photoluminescence measurements have yielded laser threshold values as low as $16.4 \mu \mathrm{W}$, nearing the transparency level of the material. Future efforts will include low-temperature nearresonance studies to examine single-QD-microdisk systems and further studies of the trade-off between microdisk size and optical loss. In addition, use of the fiber taper coupler will be examined as a tool that can greatly facilitate such experiments.

One of the authors (K.S.) thanks K. Hennessy for helpful conversations regarding AlGaAs processing. Three of the authors (K.S., T.J., and M.B.), respectively, thank the Hertz Foundation, the Charles Lee Powell Foundation, and the Moore Foundation, NPSC, and HRL Laboratories for their graduate fellowship support.

${ }^{1}$ J. Reithmaier, G. Sek, A. Loffer, C. Hoffman, S. Kuhn, S. Reitzenstein, L. Keldysh, V. Kulakovskii, T. Reinecke, and A. Forchel, Nature (London) 432, 197 (2004).

${ }^{2}$ T. Yoshie, A. Scherer, J. Hendrickson, G. Khitrova, H. Gibbs, G. Rupper, C. Ell, Q. Schenkin, and D. Deppe, Nature (London) 432, 200 (2004).

${ }^{3}$ E. Peter, P. Senellart, D. Martrou, A. Lemaître, J. Hours, J. Gérard, and J. Bloch, quant-ph/0411076 (2004).

${ }^{4}$ J. Cirac, P. Zoller, H. Kimble, and H. Mabuchi, Phys. Rev. Lett. 78, 3221 (1997).

${ }^{5}$ H. J. Kimble, Phys. Scr., T 76, 127 (1998).

${ }^{6}$ M. Bayer and A. Forchel, Phys. Rev. B 65, 041308(R) (2002).

${ }^{7}$ K. Srinivasan, P. E. Barclay, O. Painter, J. Chen, A. Y. Cho, and C. Gmachl, Appl. Phys. Lett. 83, 1915 (2003).

${ }^{8}$ Y. Akahane, T. Asano, B.-S. Song, and S. Noda, Nature (London) 425, 944 (2003).

${ }^{9}$ K. Srinivasan, P. E. Barclay, M. Borselli, and O. Painter, Phys. Rev. B 70, 081306(R) (2004).

${ }^{10}$ M. Borselli, K. Srinivasan, P. Barclay, and O. Painter, Appl. Phys. Lett. 85, 3693 (2004).

${ }^{11}$ B. Gayral, J. M. Gérard, A. Lemaître, C. Dupuis, L. Manin, and J. L. Pelouard, Appl. Phys. Lett. 75, 1908 (1999).

${ }^{12}$ P. Michler, A. Kiraz, L. Zhang, C. Becher, E. Hu, and A. Imamoglu, Appl. Phys. Lett. 77, 184 (2000).

${ }^{13}$ S. Krishna, S. Raghavan, A. Gray, A. Stintz, and K. Malloy, Appl. Phys. Lett. 80, 3898 (2002).

${ }^{14}$ G. Liu, A. Stintz, H. Li, T. Newell, A. Gray, P. Varangis, K. Malloy, and L. Lester, IEEE J. Quantum Electron. 36, 1272 (2000).

${ }^{15}$ T. Kippenberg, S. Spillane, and K. Vahala, Opt. Lett. 27, 1669 (2002).

${ }^{16}$ D. Weiss, V. Sandoghdar, J. Hare, V. Leéfevre-Seguin, J. Raimond, and S. Haroche, Opt. Lett. 22, 1835 (1995).

${ }^{17}$ Note that $V_{\text {eff }}$ for a standing wave whispering gallery mode is one-half that of a traveling wave mode. Also, our estimate of $\kappa$ assumes that $Q \sim 3.6 \times 10^{5}$ is maintained at $\lambda \sim 1.2 \mu \mathrm{m}$.

${ }^{18}$ L. Andreani, G. Panzarini and J.-M. Gérard, Phys. Rev. B 60, 13276 (1999).

${ }^{19}$ P. G. Eliseev, H. Li, G. Liu, A. Stintz, T. Newell, L. Lester, and K. Malloy, IEEE J. Sel. Top. Quantum Electron. 7, 135 (2001).

${ }^{20}$ T. Ide, T. Baba, J. Tatebayashi, S. Iwamoto, T. Nakaoka, and Y. Arakawa, Appl. Phys. Lett. 85, 1326 (2004).

${ }^{21}$ L. A. Coldren and S. W. Corzine, Diode Lasers and Photonic Integrated Circuits (Wiley, New York, 1995). 